https://doi.org/10.22319/rmcp.v11i1.4923

Revisión bibliográfica

\title{
Impacto del estrés por calor en la producción de ovinos de pelo. Revisión
}

Ricardo Vicente Pérez ${ }^{\mathrm{a}}$

Ulises Macías Cruz ${ }^{\mathrm{b} *}$

Leonel Avendaño Reyes ${ }^{b}$

Abelardo Correa-Calderón ${ }^{\mathrm{b}}$

María de los Ángeles López Baca ${ }^{\mathrm{c}}$

Ana L. Lara Rivera ${ }^{b}$

${ }^{a}$ Universidad de Guadalajara, Centro Universitario de la Costa Sur, Departamento de Producción Agrícola, Autlán de Navarro, Jal., México.

bUniversidad Autónoma de Baja California, Instituto de Ciencias Agrícolas, Valle de Mexicali, B.C., México.

${ }^{\mathrm{c}}$ Centro de Investigación en Alimentación y Desarrollo A.C., Hermosillo, Sonora, México.

*Autor de correspondencia: ulisesmacias1988@ hotmail.com

\section{Resumen:}

Frente al problema del calentamiento global y cambio climático, los pequeños rumiantes serán clave para mantener la producción de proteína de origen animal, ya que presentan superioridad para la tolerancia al calor en comparación con otros animales domésticos. Los ovinos de pelo han demostrado ampliamente su habilidad para crecer y reproducirse en escenarios naturales caracterizados por altas temperaturas y baja disponibilidad de nutrientes. La adaptación que presentan estos ovinos al estrés por calor está dada por una compleja interacción entre los mecanismos de termorregulación y la presencia de factores genéticos, lo cual les confiere plasticidad fisiológica para tolerar climas calientes sin afectar drásticamente su productividad. En México, los ovinos de pelo se encuentran distribuidos en 
los diferentes climas debido a que no presentan estacionalidad reproductiva, y esta característica permite a la industria ovina mantener una producción de carne constante a través del año; sin embargo, poca atención se ha puesto a su habilidad para producir en condiciones de estrés por calor. En este sentido, la presente revisión tiene como objetivo describir los efectos del estrés por calor sobre comportamiento reproductivo, crecimiento y termorregulación de ovinos de pelo.

Palabras clave: Borregos adaptados, Hipertermia, Homeotermos, Fertilidad de ovinos.

Recibido: 03/06/2018

Aceptado: 10/12/2018

\section{Introducción}

El principal fenómeno que amenaza la producción de alimentos de origen animal, y por consiguiente la seguridad alimentaria, es el cambio climático derivado de las emisiones de los gases con efecto invernadero ${ }^{(1)}$. El cambio climático está aumentando la temperatura ambiental y cambiando los patrones circanuales de lluvias en las diferentes regiones agroecológicas del mundo. Así, el problema de calentamiento global favorece la presencia de condiciones climáticas de estrés por calor $(\mathbf{E C})$ para los animales domésticos en regiones donde no existían, y donde de manera natural existían temperaturas altas, el EC se ha agravado a tal grado que aumentó la tasa de mortalidad de los animales ${ }^{(2)}$. Esto como una respuesta a la incapacidad del cuerpo para mantener normotermia.

En regiones áridas, semiáridas y desérticas del mundo predomina la producción de pequeños rumiantes, porque son capaces de sobrevivir en condiciones de baja disponibilidad de alimento y tienen superior tolerancia a EC comparados con los bovinos ${ }^{(1,3)}$. En ovinos, las temperaturas elevadas afectan negativamente su desarrollo y productividad, ya que disminuyen el consumo de alimento e incrementan las demandas de energía por la activación de los mecanismos de termorregulación. Los ovinos bajo EC presentan baja fertilidad, desarrollo y crecimiento fetal, así como escasa ganancia de peso y eficiencia alimenticia en la etapa de engorda ${ }^{(4,5,6)}$. Las características de la canal y calidad de la carne de ellos también son afectadas negativamente por temperaturas altas ${ }^{(3,5)}$. Cabe mencionar que el grado de los efectos del EC sobre la productividad de los ovinos depende del nivel de adaptación de cada raza, siendo las razas de pelo menos susceptibles a $\mathrm{EC}^{(7,8)}$.

Los ovinos de razas de pelo existentes en México fueron desarrollados en climas cálidos, mayormente en el continente Africano, por lo que cuentan con capacidad genética para 
tolerar y adaptarse fácilmente a climas cálidos ${ }^{(9)}$. En algunos estudios realizados en la región árida y seca del noroeste de México durante la época de verano, se ha documentado que la capacidad productiva y reproductiva de los ovinos de razas de pelo (Pelibuey, Katahdín, Dorper y sus cruzas) no se merma drásticamente durante los meses calientes de verano ${ }^{(10,11)}$. La activación de mecanismos termorregulatorios específicos de tipo fisiológico, metabólico

y endocrinológico, son responsables parcialmente de la habilidad que tienen estas razas para evitar hipertermia ${ }^{(11,12,13)}$. Además, cuentan con características fenotípicas y genotípicas que les permiten ser más tolerantes al $\mathrm{EC}^{(8)}$. En este sentido, el objetivo de esta revisión fue describir los efectos del EC sobre comportamiento reproductivo, crecimiento de corderos y termorregulación de ovinos de pelo.

\section{Cambio climático y la producción ovina}

Las regiones calientes del mundo ocupan actualmente alrededor del $50 \%$ de la superficie terrestre, proyectándose a futuro un aumento como consecuencia del calentamiento global. Además, este problema ha provocado variaciones impredecibles en la época y la cantidad de lluvia, así como disminución en la cobertura vegetal y aumento en la cantidad de áreas desérticas en el mundo, lo cual ha generado menor disponibilidad y calidad de forraje para la alimentación del ganado $^{(1)}$. Los pequeños rumiantes, como los ovinos, presentan buena adaptación a condiciones climáticas extremas comparados con otras especies domésticas, convirtiéndose en una opción de producción de alimento en regiones áridas y semi-áridas con baja disponibilidad de recursos forrajeros ${ }^{(14)}$.

Los ovinos tienen la habilidad de convertir alimentos fibrosos y de mala calidad en productos para el consumo humano (carne, leche y lana) bajo condiciones de producción precaria, donde otros animales domésticos diferentes de las cabras difícilmente podrían subsistir. Cabe mencionar que los ovinos de razas nativas de las regiones áridas y semi-áridas son los que mejor adaptación han mostrado al EC y a sobrevivir en condiciones extensivas precarias ${ }^{(3,14)}$; por consiguiente, la selección de razas apropiadas es una estrategia efectiva para mantener la producción de carne ante el escenario del cambio climático ${ }^{(1)}$.

En México, los ovinos de raza de pelo han mostrado tolerar adecuadamente condiciones climáticas de EC en regiones agroecológicas calientes. Las temperaturas altas en estas regiones no han sido un factor que contribuya drásticamente a disminuir la capacidad reproductiva $^{(12,15,16)}$ y el crecimiento de las crías $^{(5)}$. Así que los ovinos de pelo son razas adaptadas a climas cálidos, con una plasticidad fisiológica y metabólica que les permite tolerar este tipo de ambientes sin comprometer su productividad ${ }^{(13)}$. 


\section{Estrés por calor en ovinos de pelo}

La disminución en el consumo de alimento y la activación del eje hipotálamo-hipófisisadrenal como consecuencia del EC, produce en los ovinos una disminución en el comportamiento productivo y reproductivo, y por ende, en la productividad del rebaño ${ }^{(4,17)}$. Sin embargo, estas alteraciones no son tan marcadas en los ovinos de pelo como pudieran presentarse en razas de lana ${ }^{(5,12)}$.

\section{Efectos en la reproducción}

La fertilidad en las ovejas de pelo parece ser más afectada por el fotoperiodo y otras señales nutricionales que por las mismas temperaturas ambientales elevadas. Algunos estudios reportan que la conducta de estro y la ovulación no es alterada por el EC de verano ${ }^{(12,15,16)}$. No obstante, la funcionalidad del cuerpo lúteo (en base a niveles de progesterona sanguínea) mostró ser disminuida por efecto del EC agudo ${ }^{(18)}$ y crónico $^{(12,15)}$. Es posible que esta disminución de progesterona se deba a la regresión prematura de los cuerpos lúteos, tal como Tabárez-Rojas et al ${ }^{(18)}$ lo observaron en ovejas Pelibuey después de estar sujetas a $37 \pm 2.5$ ${ }^{\circ} \mathrm{C}$ en una cámara termo-ambiental. Cabe mencionar que en ese estudio el desarrollo embrionario temprano fue inalterado por el EC inducido ${ }^{(18)}$.

Hasta el momento no se ha determinado el mecanismo por el cual las ovejas de pelo mantienen su actividad reproductiva y fertilidad en condiciones de hipertermia. El EC reduce la función reproductiva debido a la activación del eje hipotálamo-hipófisis-adrenal (eje del estrés), el cual suprime la función del eje hipotálamo-hipófisis-gonadal (eje reproductivo) ${ }^{(1)}$. El eje del estrés promueve la síntesis y liberación de cortisol en las glándulas adrenales, y esta hormona inhibe a nivel de hipotálamo la producción de la hormona liberadora de gonadotropinas $(\mathrm{GnRH})^{(17)}$. La GnRH es necesaria para estimular la síntesis y liberación de la hormona folículo estimulante (FSH) y la hormona luteinizante (LH) en la hipófisis, y ambas hormonas hipofisiarias son necesarias para producir y liberar óvulos fértiles ${ }^{(19)}$. Dado que cortisol también se incrementa en ovinos de pelo en respuesta al $\mathrm{EC}^{(7)}$, se podrían plantear dos posibles hipótesis que explican la ausencia del efecto del EC sobre la fertilidad de ovejas de pelo: 1) una menor sensibilidad del eje reproductivo en reflejo al aumento del cortisol, y 2) el aumento en los niveles de cortisol debido a EC no son suficientes para afectar negativamente la funcionalidad del eje reproductivo.

Por otra parte, el EC parece alterar negativamente el desarrollo pre- y post-natal de las crías en ovinos de pelo. La hipertermia gestacional puede reducir el desarrollo y crecimiento placental, promoviendo una disminución en la transferencia de nutrientes feto-maternal ${ }^{(11)}$. Esto genera un problema de retardo en el crecimiento fetal y, consecuentemente, el nacimiento de corderos con peso ligero, débiles y con altas posibilidad de muerte perinatal ${ }^{(20)}$. 
Vicente-Pérez et $a l^{(6)}$ también reportaron que el EC durante el último tercio de gestación no afectó el peso al nacimiento de los corderos, pero sí redujo la prolificidad y el peso de la camada al nacimiento en ovinos de pelo, comparado con condiciones termoneutrales de invierno. Dado que sincronizaron con progestágenos y gonadotropina coriónica equina para aumentar el porcentaje de partos dobles en ese estudio, la menor prolificidad en verano pudo ser un reflejo de una reabsorción fetal a consecuencia de las temperaturas altas. En este sentido, no se recomienda programar partos durante los meses más calientes en regiones cálidas.

\section{Efectos en el crecimiento de los corderos}

Bajo condiciones de México, la información disponible en relación al impacto que tiene el EC en el crecimiento y desarrollo de los corderos de raza de pelo es escasa. La temperatura ambiental es un factor que tiene cierto control sobre la capacidad de los animales para consumir alimento $^{(4)}$, y el EC conduce a una reducción en el consumo de materia seca, un aumento en el consumo de agua y a una necesidad extra de energía metabolizable para la activación de los mecanismos de termorregulación en los $\operatorname{corderos}^{(1,4)}$. Lo anterior contribuye a que los requerimientos de energía de mantenimiento aumenten bajo un escenario corporal donde la entrada de energía a través de la ingesta de alimento se reduce ${ }^{(1,14)}$, de esta manera, los corderos de engorda detienen la tasa de crecimiento y reducen su eficiencia alimenticia. En casos extremos, principalmente observado en razas no adaptadas, el balance energético se vuelve negativo siendo incosteable esta actividad en climas calientes ${ }^{(2)}$.

En un estudio hecho en corderas Dorper x Pelibuey, se encontró que las condiciones de EC redujeron la tasa de crecimiento y la eficiencia alimenticia en 28 y $20 \%$, respectivamente ${ }^{(5)}$. Comparando dos trabajos realizados en corderos Dorper x Pelibuey en un mismo sitio de estudio, uno en $\mathrm{EC}^{(21)}$ y el otro en condiciones termoneutrales ${ }^{(22)}$, se observó menor ganancia diaria de peso (230 vs $280 \mathrm{~g} /$ día) y consumo de materia seca ( $1.0 v s 1.3 \mathrm{~kg} / \mathrm{d}$ )ía, sin aparente diferencia en la eficiencia alimenticia (216 vs. $220 \mathrm{~g} / \mathrm{kg}$ de materia seca), en el estudio realizado en EC. Este efecto negativo del EC en el comportamiento productivo de ovinos de engorda se asoció con un aumento en el gasto energético en el proceso de termorregulación. Condiciones de hipertermia en ovinos pueden aumentar los requerimientos nutricionales de mantenimiento entre 10 y $20 \%{ }^{(23)}$.

\section{Termorregulación en ovinos de pelo}

La activación de mecanismos compensatorios y adaptativos permiten a los ovinos de pelo ser eficientes para tolerar temperaturas mayores al límite superior de su zona termoneutral, esto sin comprometer drásticamente su productividad. Cabe mencionar que la zona termoneutral para ovinos en general ha sido ubicada entre 12 y $27^{\circ} \mathrm{C}^{(1,4)}$, Neves $e$ e $a l^{(24)}$ sugieren que el 
límite superior de esta zona puede ser considerada de $30^{\circ} \mathrm{C}$ en el caso específico de las razas de pelo. Esto último evidencia que los ovinos de razas de pelo, a diferencia de las razas de lana, toleran de manera natural temperaturas más elevadas.

El tipo de EC al cual se exponen los ovinos se evalúa generalmente en base al índice temperatura-humedad (ITH). En general, un ovino puede comenzar a experimentar EC a ITH $>72$ unidades $^{(25)}$, no obstante, en una revisión publicada por Marai et $\mathrm{l}^{(4)}$ se señala que el EC se presenta a partir de las 82 unidades, pudiéndose clasificar basándose en el ITH como moderado ( 82 a $<84)$, severo $(\geq 84$ a $<86)$ y muy severo $(\geq 86)$. Sin embargo, esta información debe tomarse con precaución, ya que Neves et $a l^{(24)}$ encontraron que las ovejas de pelo comienzan a presentar signos de EC cuando el ITH alcanza entre 78 y 79 unidades. Los ovinos de pelo toleran temperaturas mayores que ovinos de lana, por consiguiente, es de esperar que el ITH donde cualquier raza ovina comienza a experimentar síntomas de EC se ubique por debajo de las 79 unidades, y no de las 82 unidades. Aunque, se requiere investigar con precisión el punto de inflexión donde los ovinos presentan EC, tal como está establecido en otras especies.

La mayor tolerancia que presentan los ovinos de pelo a condiciones de EC es el resultado de adaptaciones genéticas y fenotípicas, así como a la activación de mecanismos fisiológicos, metabólicos y endocrinológicos, los cuales ayudan a mantener un balance de agua corporal adecuado y condiciones de normotermia $\left(38.3\right.$ a $\left.39.9^{\circ} \mathrm{C}\right)$ a un costo energético bajo ${ }^{(5,13)}$. Cabe mencionar que varios de los mecanismos activados por los ovinos de pelo también son activados por los de lana, pero estos últimos siempre presentan mayor incremento en su temperatura corporal conforme aumenta la temperatura ambiental ${ }^{(26)}$.

\section{Adaptaciones genéticas}

Genéticamente, los ovinos de las razas de pelo han mostrado mayor superioridad para tolerar temperaturas ambientales altas que la mayoría de las razas de lana ${ }^{(8)}$. Por ejemplo, las ovejas de raza Blackbelly mostraron menor temperatura rectal (TR) y frecuencia respiratoria (FR) que las ovejas de lana Dorset en condiciones de $\mathrm{EC}^{(20)}$. Similarmente, ovinos Pelibuey tuvieron mayor capacidad termorregulatoria que ovinos Suffolk bajo EC agudo $\left(37 \pm 2.5^{\circ} \mathrm{C}\right.$ durante $6 \mathrm{~h} / \mathrm{día})^{(18)}$. Esta variabilidad genética está asociada con la portabilidad de genes de termo-tolerancia, los cuales han sido poco estudiados en ovinos de pelo. Un estudio demostró que estos ovinos toleraran más el EC porque activan genes de termo-tolerancia asociados a la expresión de las proteínas del "choque térmico" (HSP por sus siglas en inglés) ${ }^{(26)}$. En condiciones de EC, las HSP confieren protección a las células para evitar su apoptosis, y por consiguiente, son parcialmente responsables del proceso de adaptación de los ovinos de pelo $^{(27)}$. 
Un estudio reportó que las ovejas Pelibuey, además de presentar menor TR y FR, tuvieron mayor concentración de HSP70 (2.86 vs $0.53 \mathrm{ng} / \mathrm{ml}$ ) respecto a ovejas Suffolk cuando fueron expuestas a EC dentro de una cámara climática ${ }^{(26)}$. La reducida expresión de HSP70 en ovejas Suffolk fue asociada con una disminución en la viabilidad de las células mononucleares sanguíneas cultivadas in vitro, y consecuentemente, con una menor adaptación a climas calientes. Es preciso señalar que la HSP70 es el marcador genético mayormente expresado en razas de ovinos y cabras adaptadas a condiciones ambientales de $\mathrm{EC}^{(26,27,28)}$. No obstante, se debe tomar en cuenta que, si bien en ovinos de pelo solamente se han logrado detectar los genes ligados con la síntesis de la HSP70, en la actualidad ya se cuenta con la identificación de varios genes asociados con la termo-tolerancia de pequeños rumiantes adaptadas al EC. Es posible que los ovinos de pelo también sean portadores de algunos de estos genes, sin embargo, se requiere confirmar esta hipótesis.

En ovinos nativos del desierto se han identificado genes de termo-tolerancia asociados con coloración y pigmentación de la piel (FGF2, GNA13, PLCB1), metabolismo energético y digestivo (MYH, TRHDE, ALDH1A3 y GPR50), y respuesta inmune ${ }^{(9,28,29)}$. Recientemente, en razas de la India adaptadas al EC también se encontraron algunas mutaciones en los polimorfismos G1270A y C888T ligados al gen GPR50, las cuales se asociaron con una mejor tolerancia térmica ${ }^{(29)}$. Por su parte, Alamer ${ }^{(30)}$ menciona que la expresión de genes ligados a la prolactina afecta el mantenimiento del volumen del fluido extracelular, consumo de agua, regulación de las glándulas sudoríparas y crecimiento estacional del pelo en ovinos. En general, esto podría explicar porque esos ovinos adaptados al EC, y posiblemente también los ovinos de pelo, son eficientes en el uso de la energía metabolizable y agua, así como en mantener condiciones de homeotermia a un mínimo costo energético en escenarios de temperaturas altas.

Cabe mencionar que considerando la presencia de genes de termo-tolerancia en ovinos adaptados, la selección genética asistida a través de marcadores genéticos podría ser una herramienta para distinguir ovinos sobresalientes para tolerancia y adaptación al EC. En algunos países del mediterráneo ya se está investigando y llevando a cabo la selección por marcadores genéticos ligados con la termo-tolerancia en pequeños rumiantes ${ }^{(31)}$. Hasta el momento, el conocimiento sobre termo-tolerancia en ovinos de pelo tiene importantes avances en cuanto a las adaptaciones fenotípicas y los mecanismos fisiológicos, endocrinológicos y bioquímicos de termorregulación, los cuales son marcadores biológicos que también deben ser considerados en la selección de individuos termo-tolerantes. 


\section{Adaptaciones fenotípicas}

Los ovinos de pelo poseen características fenotípicas que les confieren adaptabilidad al EC ${ }^{(9)}$. La presencia de pelo en lugar de lana otorga a estas razas una ventaja en cuanto a la pérdida de calor, tanto por medios no evaporativos como evaporativos ${ }^{(7,13)}$. Por una parte, el pelo, al ser delgado y corto, facilita el flujo de aire hacia la piel, permitiendo que el calor acumulado en la superficie corporal se transfiera al ambiente por radiación o convección ${ }^{(7)}$; o bien, a través del sudor en una forma más eficiente ${ }^{(8)}$. Contrariamente, la lana funciona como un aislante impidiendo la entrada y salida de calor al cuerpo, al mismo tiempo que no permite la penetración del aire hasta la piel. Esto trae como consecuencia que los mecanismos de disipación de calor corporal de tipo no evaporativos y la sudoración sean ineficaces para regular la temperatura corporal en razas lanares ${ }^{(32,33)}$. Además, el número de glándulas sudoríparas así como el área que ocupan es mayor en las razas de pelo que en las de lana, por lo cual la tasa de sudoración como mecanismo de disipación de calor corporal es mejor en razas de pelo ${ }^{(8)}$. El espesor de la piel es otro factor fenotípico que causa diferencias entre las razas en la capacidad termorreguladora; los ovinos de pelo tienen piel más delgada comparados con los ovinos de lana, característica que favorece la disipación (radiación y sudoración) de calor interno a través de la piel ${ }^{(33)}$.

Por otro lado, el color del pelo juega un papel crucial en la capacidad que tienen los ovinos deslanados para transferir el exceso de calor corporal hacia el ambiente o viceversa ${ }^{(8,33)}$. La presencia de colores claros es benéfico para el confort de los ovinos de pelo porque presentan menor tasa cardiaca, FR y TR en comparación con ovinos de pelo color obscuro ${ }^{(34)}$. Esto se debe a que el color claro refleja la radiación solar, mientras que el color oscuro la absorbe, provocando una mayor acumulación de calor corporal en animales de pelo oscuro ${ }^{(8,32,34)}$. Por lo tanto, además de tener pelo, las características propias del mismo y la piel confieren a los ovinos de pelo una mejor adaptación a climas calientes.

\section{Mecanismos fisiológicos}

Cuando los ovinos se encuentran en rangos de temperatura ambiental de confort térmico realizan un esfuerzo mínimo para mantener normotermia ${ }^{(35)}$. Sin embargo, este equilibrio homeostático se compromete al incrementarse la temperatura ambiental por encima del límite superior de la zona termoneutral ${ }^{(3)}$. En dichas condiciones, los ovinos requieren realizar en un primer plano ajustes fisiológicos para tratar de disipar el exceso de carga de calor corporal $^{(35)}$; pero de no ser suficiente, mecanismos endocrinológicos y metabólicos de termorregulación pueden también ser activados. Por lo tanto, es común observar las siguientes respuestas fisiológicas de termorregulación en ovinos de pelo que sufren EC: mayor FR y consumo de agua pero menor consumo de alimento. 
Cabe mencionar que el aumento en la FR es el principal mecanismo que ayuda a evitar la hipertermia en ovinos estresados por calor ${ }^{(32,33)}$. Marai et $a l^{(4)}$ mencionan que, independientemente de la raza, los ovinos bajo EC disipan cuando menos el $60 \%$ de la carga de calor corporal vía tracto respiratorio. De tal manera que, cuando se presentan condiciones de temperaturas altas y aumenta la TR, paralelamente se da un incremento en la $\mathrm{FR}^{(3,13)}$. En este sentido, el aumento en TR, FR y otras constantes fisiológicas debido a EC ocurre naturalmente en todas razas ovinas ${ }^{(12,13,20,26)}$. Sin embargo, los aumentos en los valores promedios de estas variables fisiológicas son menores en los ovinos de pelo que en los ovinos de lana ${ }^{(20,26,33)}$. Esto sugiere que las razas de pelo posiblemente realizan, simultáneamente con el incremento de la FR, otro tipo de ajustes fisiológicos, o bien, ajustes de otra naturaleza (p.e. reducción de su actividad motora o actividad metabólica) ${ }^{(20,32,35)}$.

Algunos estudios indican que la menor FR observada en los ovinos de pelo puede estar relacionada con una continua pérdida de calor corporal a través de la piel en ambientes de $\mathrm{EC}^{(32,33)}$. De esta manera, el esfuerzo por disipar calor resulta en vasodilatación y la redistribución del flujo sanguíneo hacia los tejidos periféricos para incrementar la sensibilidad de la piel y promover la pérdida de calor vía radiación, convección y sudoración $^{(4,36)}$. La evaporación de agua a través de la piel por efecto de sudación ( 10\%) en baja en ovinos de pelo, por lo que las pérdidas de calor a través del tracto respiratorio son las más importantes bajo condiciones calientes ${ }^{(36)}$, tal como previamente fue mencionado (60 a $90 \%)^{(37)}$. Así, el aumento en la FR y las pérdidas de calor a través de la piel funcionan en forma sinérgica para hacer más eficiente la termorregulación en los ovinos de pelo ${ }^{(13)}$.

Por otro lado, se ha observado que, bajo un clima árido seco, los patrones circadianos de TR, FR y temperatura de capa de pelo en las diferentes regiones corporales cambian acorde a la temperatura ambiental durante primavera (termoneutral); pero en verano bajo EC, las temperaturas de capa de pelo fluctúan con la temperatura ambiental mientras que la FR como las pérdidas de calor a través de la piel son insuficientes ${ }^{(13)}$. Este ritmo circadiano en la FR bajo condiciones naturales de temperaturas altas, sugiere ser un mecanismo adaptativo fisiológico desarrollado por los ovinos de pelo para mantener homeotermia sin comprometer su hidratación. Recientemente, otro estudio realizado durante el verano en la región árida del noroeste de México, señala que, en el mes más caliente (agosto), los ovinos de pelo desarrollaron heterotermia adaptativa ${ }^{(38)}$; un mecanismo que usualmente tienen los animales homeotermos adaptados al desierto ${ }^{(39)}$. El mecanismo de heterotermia adaptativa permite a los animales tolerar una mayor carga de calor corporal, la cual es disipada principalmente por un aumento drástico en la $\mathrm{FR}$ en horarios donde es mínima o inexistente la radiación solar ${ }^{(35,39)}$. Este mecanismo previene la deshidratación en los animales homeotermos del desierto durante las épocas calientes del año ${ }^{(39)}$. 
En respuesta al incremento en la FR y la sudoración, también crece el consumo de agua como mecanismo de enfriamiento y una forma de compensar el déficit hídrico, dado el aumento en la cantidad de vapor de agua que sale a través del tracto respiratorio ${ }^{(4,6,40)}$ y la sudoración ${ }^{(8,36)}$. También se observa como mecanismo de termorregulación una marcada reducción en el consumo y digestión del alimento ${ }^{(4,6,7,40)}$. Marai et $a l^{(4)}$ sugieren que la reducción en el consumo de alimento se regula a nivel endócrino y tiene como objetivo reducir la producción endógena de calor metabólico.

\section{Mecanismos endocrinológicos}

Las respuestas fisiológicas y de comportamiento que se desencadenan en los animales estresados por calor son reguladas a nivel neuroendocrino ${ }^{(17,41)}$. En condiciones de EC es común observar una reducción en las concentraciones sanguíneas de hormonas tiroideas (T3 [triyodotironina] y T4 [tiroxina]) como un mecanismo para disminuir la producción de calor metabólico, ya que estas hormonas son encargadas de mediar el metabolismo animal ${ }^{(39)}$. Esto no ha sido la excepción en ovinos de pelo, y un estudio donde usaron una cámara climática para inducir EC en ovejas preñadas de las razas Blackbelly y Dorset, encontraron que las concentraciones de T3 y T4 disminuyeron en ambas razas a una temperatura de $33.8^{\circ} \mathrm{C}$; no obstante, las ovejas de pelo presentaron un menor cambio en las concentraciones de las hormonas ${ }^{(20)}$. Esto sugiere que los ovinos de pelo son capaces de mantener su metabolismo en un mejor equilibrio frente al insulto térmico.

El efecto del EC sobre los niveles de las hormonas tiroideas en ovinos de pelo puede deberse a un aumento en la síntesis hipotalámica del factor inhibidor de la tirosina (FIT) ${ }^{(1)}$. El EC estimula los receptores térmicos periféricos, los cuales a su vez suprimen al centro del apetito en el hipotálamo, provocando una mayor síntesis y liberación del FIT. Este factor reduce la liberación de la hormona estimulante de la tiroides, y consecuentemente, afecta negativamente la producción hormonal en la glándula tiroidea ${ }^{(3,41)}$. Cabe destacar que la liberación de T4 presenta mayor sensibilidad al EC comparado con la liberación de T3 ${ }^{(42)}$. Esto sugiere que la tiroxina está más asociada a la reducción del consumo de alimento y a su vez, a la disminución endógena de calor metabólico.

El cortisol es otra hormona que juega un papel muy importante en el proceso de adaptación a la presencia de factores estresantes ${ }^{(17,41)}$. Este glucocorticoide es un mediador de la gluconeogénesis hepática; la disponibilidad de la glucosa en el organismo del animal es esencial durante un estado de alarma o estrés, ya que funciona como una fuente de energía de rápida disponibilidad celular ${ }^{(41,42,43)}$. En ovinos de razas de pelo ${ }^{(7)}$, así como en otras razas

no encontradas en México pero adaptadas al EC tal como la Malpura en la India ${ }^{(40,42)}$, se ha encontrado elevado cortisol sérico en respuesta al EC. Esto obedece a una necesidad del organismo por tener energía disponible para hacer frente al gasto energético extra que implica 
la activación de los mecanismos de termorregulación de tipo evaporativo. Así, el aumento del cortisol se relaciona con una mayor glucosa en la circulación sanguínea producto de la activación del metabolismo celular hepático ${ }^{(3)}$, y a su vez, con un aumento en la liberación de colesterol, metabolito sanguíneo que por acción enzimática es convertido a cortisol en la glándula adrenal ${ }^{(17,41)}$.

La insulina también es una hormona metabólica que tiene un rol importante en la regulación del metabolismo energético bajo condiciones de EC en ovinos ${ }^{(44)}$. Los niveles de esta hormona aumentan en respuesta al EC produciendo un cuadro de hiperinsulinemia, y se intuye que es una estrategia para proteger el correcto funcionamiento pancreático y favorecer una mayor producción de $\mathrm{HSP}^{(45)}$. Así, mientras que el EC reduce el consumo de alimento, la hiperinsulinemia evitan la lipólisis y el aumento en las concentraciones de ácidos grasos no esterificados, los cuales en exceso provocan apoptosis de las células pancreáticas $\beta^{(46)}$. Cabe mencionar que este cuadro de hiperinsulinemia provocado por el EC resulta favorable para mantener el peso vivo, la condición corporal y, por lo menos, una mínima ganancia de peso, ya que aun cuando el consumo de alimento se reduce, la insulina evita que se utilicen las reservas corporales ${ }^{(47)}$. Al respecto, un estudio realizado en ovinos Afshari (raza adaptada a EC) reportó una reducción en los requerimientos de mantenimiento bajo condiciones de EC severo, ya que los ovinos siguieron ganando peso aún y cuando el consumo de alimento se redujo en $17.5 \%{ }^{(44)}$. Los autores concluyeron que este efecto positivo del EC en razas adaptadas podría estar relacionado a una alteración en el metabolismo postabsortivo generada por la elevación en las concentraciones sanguíneas de insulina ${ }^{(44,45)}$. En ovinos de pelo no se ha estudiado el efecto del EC sobre la secreción de insulina, sin embargo, algunos estudios sugieren que puede presentarse un similar ajuste metabólico ${ }^{(5,12,15,38)}$. Esto explicaría parcialmente el hecho que estos ovinos siguen creciendo bajo EC.

La epinefrina y norepinefrina son otras sustancias que han demostrado intervenir como hormonas o neurotransmisores en el proceso de termorregulación de los ovinos, sin embargo, su actividad en ovinos de pelo estresados por calor no ha sido estudiada. Se conoce que, en animales sometidos a EC, la epinefrina y la norepinefrina activan la función cardiovascular para suministrar suficiente sangre a los órganos vitales ${ }^{(43,48)}$. La epinefrina también se relaciona con la gluconeogénesis hepática y la lipolisis, procesos metabólicos necesarios para el suministro de energía a los sistemas encargados de la termorregulación ${ }^{(49)}$.

\section{Mecanismos bioquímicos}

La activación de ajustes fisiológicos en ovinos de pelo para mantener normotermia en climas cálidos, está estrechamente relacionada con cambios en los niveles de analitos sanguíneos. Igualmente, los cambios en algunos analitos sanguíneos pueden directamente ser ocasionados por el EC, ya sea como un reflejo de la capacidad de adaptación o falta de la $\operatorname{misma}^{(50)}$. 
Un estudio reportó que las concentraciones séricas de glucosa, colesterol y triglicéridos en corderas Dorper x Pelibuey disminuyeron por efecto del EC crónico al que son sometidas durante la época de verano en una región desértica del noroeste de México ${ }^{(13)}$. Considerando que en ese estudio encontraron un aumento de más del $100 \%$ en la FR con respecto a la observada en primavera (época termoneutral), los autores asociaron esa disminución en los metabolitos a un elevado gasto energético que tienen los músculos del tracto respiratorio con el aumento en la FR. En ese mismo estudio se detectó un aumento en los niveles séricos de urea y una disminución en los de potasio sin afectar las concentraciones de sodio; situación que indica que no hubo pérdidas de agua corporal por orina, heces y sudoración. Esto sugiere que los ovinos de pelo cuentan con mecanismos adaptativos metabólicos que reducen las posibilidades de un cuadro de deshidratación. En otro estudio hecho en la misma región desértica de México ${ }^{(38)}$, se demostró que los ovinos de pelo activan un metabolismo postabsortivo energético cuando el EC es de tipo crónico e intenso, mientras que, en condiciones de EC agudo, la glucosa es la principal fuente de energía para el gasto energético que implica el aumento en la FR. Así, los niveles sanguíneos altos de glucosa como respuesta al EC agudo pueden ser explicados por el aumento en los niveles de cortisol, hormona que estimula la gluconeogénesis para proveer glucosa como combustible energético a las células ${ }^{(44)}$.

Cabe mencionar que los efectos del EC sobre la concentración de compuestos sanguíneos son muy variables a través de los estudios, por lo que en ocasiones se vuelve complicado explicar los ajustes metabólicos que realizan los ovinos de pelo para sobrevivir y adaptarse a climas cálidos. Por lo anterior, factores como raza, edad, tipo de EC, nutrición, estado fisiológico y otros, deben ser considerados al momento de la interpretación de resultados. Recientemente se demostró que la falta de sombra en los corrales promueve un aumento en las concentraciones de metabolitos relacionados con el metabolismo energético y lipídico, no así en el metabolismo de proteínas, en corderas de la cruza Dorper x Pelibuey estresadas por calor $^{(50)}$. En ese mismo estudio se observó un aumento en las concentraciones séricas de los electrolitos sodio y cloro, lo cual se atribuyó a un mayor consumo de agua. En otro estudio se encontró que la edad y la lactación son dos factores que alteran las concentraciones sanguíneas de glucosa, colesterol y urea, pero no las concentraciones de triglicéridos, proteína total y electrolitos en sangre de ovinos hembras de la cruza Dorper x Pelibuey mantenidos en condiciones de EC natural ${ }^{(51)}$. Las corderas de destete y las ovejas lactando presentan menores concentraciones glucosa y colesterol comparados con ovejas nulíparas y multíparas no lactando. Mientras que los resultados de corderas se relacionaron con una mayor FR, lo observado en ovejas lactantes se atribuyó al efecto de una redistribución de nutrientes para la síntesis de los compuestos lactosa y grasa de la leche. Usando ovinos de similar genotipo que en los estudios previamente descritos, una investigación determinó que el ambiente uterino en el que se desarrollan los corderos durante el último tercio de gestación, no es un factor que repercuta en la variación de las concentraciones de metabolitos y 
electrolitos sanguíneo de corderos estresados por calor durante el periodo de engorda ${ }^{(52)}$. La restricción nutricional también mostró tener poco efecto en las concentraciones de metabolitos sanguíneos relacionados con el metabolismo energético en ovejas de preñez tardía mantenidas en un ambiente caliente ${ }^{(11)}$.

\section{Conclusiones e implicaciones}

Los ovinos de razas de pelo se caracterizan por ser rústicos y adaptarse con facilidad a diferentes condiciones de producción, incluyendo aquellas donde el clima predominante es de calor extremo y la calidad de los forrajes deficiente. Al parecer, las razas de pelo tienen la capacidad para crecer y reproducirse en condiciones de EC porque cuentan con genes de termo-tolerancia, además, fenotípicamente, la estructura de su piel y pelo les da ventajas frente a las razas de lana para disipar el calor corporal de una manera más eficiente por vías evaporativas o no evaporativas. Los patrones circadianos de la FR, así como las características de piel y ajustes metabólicos, permiten a los ovinos de pelo disminuir la carga de calor corporal en forma eficiente $y$, posiblemente, a un menor costo energético comparado con las razas de lana. Finalmente, se destaca que, frente al problema del calentamiento global y cambio climático, la selección de razas tolerantes al calor y eficientes en el uso de nutrientes será una necesidad para garantizar la producción de proteínas de origen animal.

\section{Literatura citada:}

1. Sejian V, Bhatta R, Gaughan J, Malik PK, Naqvi SMK, Lal R. Adapting sheep production to climate change. In: Sheep production adapting to climate change. Singapore: Springer Singapore; 2017:1-29.

2. Rojas-Downing MM, Nejadhashemi AP, Harrigan T, Woznicki SA. Climate change and livestock: Impacts, adaptation, and mitigation. Clim Risk Manage 2017;16:145-163.

3. Al-Dawood A. Towards heat stress management in small ruminants- a review. Ann Anim Sci 2017;17:59-88.

4. Marai IFM, El-Darawany AA, Fadiel A, Abdel-Hafez MAM. Physiological traits as affected by heat stress in sheep-A review. Small Ruminant Res 2007;71(1-3):1-12.

5. Macías-Cruz U, Avendaño-Reyes L, Álvarez-Valenzuela FD, Torrentera- Olivera NG, Meza-Herrea CA, Mellado-Bosque M, et al. Crecimiento y características de canal en corderas tratadas con clorhidrato de zilpaterol durante primavera y verano. Rev Mex Cienc Pecu 2013;4(1):1-12. 
6. Vicente-Pérez R, Avendaño-Reyes L, Álvarez F, Correa-Calderón A, Meza-Herrera CA, Mellado M, et al. Comportamiento productivo, consumo de nutrientes y productividad al parto de ovejas de pelo suplementadas con energía en el preparto durante verano e invierno. Arch Med Vet 2015;47(3):301-309.

7. Correa MPC, Dallago BSL, Paiva SR, Canozzi ME, Louvandini H, Barcellos JJ, et al. Multivariate analysis of heat tolerance characteristics in Santa Inês and crossbred lambs in the Federal District of Brazil. Trop Anim Health Pro 2013;45(6):1407-1414.

8. McManus C, Louvandini H, Gugel R, Sasaki LC, Bianchini E, et al. Skin and coat traits in sheep in Brazil and their relation with heat tolerance. Trop Anim Health Pro 2011;43(1):121-126.

9. Aguilar-Martinez CU, Berruecos-Villalobos JM, Espinoza-Gutiérrez B, Segura-Correa JC, Valencia-Méndez J, Roldán-Roldán A. Origen, historia y situacion actual de la oveja pelibuey en Mexico. Trop Subtrop Agroecosyst 2017;20(3):429-439.

10. Macías-Cruz U, Álvarez-Valenzuela FD, Rodríguez-García J, Correa-Calderón A, Torrentera-Olivera NG, Molina-Ramírez L, et al. Growth and carcass traits in pure pelibuey lambs and crosses F1 with dorper and katahdin breeds in confinement. Arch Med Vet 2010;42(3):147-154.

11. Macías-Cruz U, Álvarez-Valenzuela FD, Correa-Calderón A, Díaz-Molina R, Mellado M, Meza-Herrera CA, et al. Thermoregulation of nutrient-restricted hair ewes subjected to heat stress during late pregnancy. J Therm Biol 2013;38(1):1-9.

12. Macías-Cruz U, Gastélum MA, Álvarez FD, Correa A, Díaz R, Meza-Herrera CA, et al. Effects of summer heat stress on physiological variables, ovulation and progesterone secretion in Pelibuey ewes under natural outdoor conditions in an arid region. Anim Sci J 2016;87(3):354-360.

13. Macías-Cruz U, López-Baca MA, Vicente R, Mejia A, Álvarez FD, Correa-Calderón A, et al. Effects of seasonal ambient heat stress (spring vs. summer) on physiological and metabolic variables in hair sheep located in an arid region. Int $\mathrm{J}$ Biometeorol 2016;60(8):1279-1286.

14. Shinde AK, Sejian V. Sheep husbandry under changing climate scenario in India: An overview. Indian J Anim Sci 2013;83(10):998-1008.

15. Macías-Cruz U, Sánchez-Estrada TJ, Gastélum-Delgado MÁ, Avendaño-Reyes L, Correa-Calderón A, Álvarez-Valenzuela FD, et al. Seasonal reproductive activity of Pelibuey ewes under arid conditions of Mexico. Arch Med Vet 2015;47(3):381-386. 
16. Gastelum-Delgado MA, Avendaño-Reyes L, Álvarez-Valenzuela FD, Correa-Calderón A, Meza-Herrera CA, Mellado M, et al. Conducta estral circanual en ovejas Pelibuey bajo condiciones áridas del noroeste de México. Rev Mex Cienc Pecu 2015;6(1):109118.

17. Tabarez-Rojas A, Porras-Almeraya A, Vaquera-Huerta H, Hernández-Ignacio J, Valencia J, Rojas-Maya S, et al. Desarrollo embrionario en ovejas pelibuey y suffolk en condiciones de estrés calórico. Agrociencia 2009;43(7):671-680.

18. Smith SM, Vale WW. The role of the hypothalamic-pituitary-adrenal axis in neuroendocrine responses to stress. Dialogues Clin Neurosci 2006;8(4):383-395.

19. Ralph CR, Lehman MN, Goodman RL, Tilbrook AJ. Impact of psychosocial stress on gonadotrophins and sexual behaviour in females: Role for cortisol? Reproduction 2016;152(1):R1-R14.

20. Ross TT, Goode L, Linnerud AC. Effects of high ambient temperature on respiration rate, rectal temperature, fetal development and thyrold gland activity in tropical and temperate breeds of sheep. Theriogenology 1985;24(2):259-269.

21. Dávila-Ramírez JL, Macías-Cruz U, Torrentera-Olivera NG, González-Ríos H, SotoNavarro SA, Rojo-Rubio R, et al. Effects of zilpaterol hydrochloride and soybean oil supplementation on feedlot performance and carcass characteristics of hair-breed ram lambs under heat stress conditions. J Anim Sci 2014;92(3):1184-1192.

22. Macías-Cruz U, Álvarez-Valenzuela FD, Soto-Navarro SA, Águila-Tepato E, Avendaño-Reyes L. Effect of zilpaterol hydrochloride on feedlot performance, nutrient intake, and digestibility in hair-breed sheep. J Anim Sci 2013;91(4):1844-1849.

23. NRC. Nutrient requirements of small ruminants: Sheep, goats, cervids, and new world camelids; 2007.

24. Neves MLMW, De Azevedo M, Da Costa LAB, Guim A, Leite AM, Chagas JC. Níveis críticos do Índice de Conforto Térmico para ovinos da raça Santa Inês criados a pasto no agreste do Estado de Pernambuco. Acta Sci Anim Sci 2009;31(2):167-175.

25. López R, Pinto-Santini L, Perozo D, Pineda J, Oliveros I, Chácon T, et al. Confort térmico y crecimiento de corderas West African pastoreando con y sin acceso a sombra artificial. Arch Zootec 2015;64(246):139-146.

26. Romero RD, Montero A, Montaldo HH, Rodríguez AD, Hernández Cerón J. Differences in body temperature, cell viability, and HSP-70 concentrations between Pelibuey and Suffolk sheep under heat stress. Trop Anim Health Pro 2013;45(8):1691-1696. 
27. Singh KM, Singh S, Ganguly I, Nachiappan RK, Ganguly A, Venkataramanan R, et al. Association of heat stress protein 90 and 70 gene polymorphism with adaptability traits in Indian sheep (Ovis aries). Cell Stress Chaperon 2017;22(5):675-684.

28. Rout PK, Kaushik R, Ramachandran N. Differential expression pattern of heat shock protein 70 gene in tissues and heat stress phenotypes in goats during peak heat stress period. Cell Stress Chaperon 2016;21(4):645-651.

29. Saxena VK, Kumar D, Naqvi SMK. Molecular characterization of GPR50 gene and study of its comparative genetic variability in sheep breeds adapted to different thermocontrasting climatic regimens. Int J Biometeorol 2017;61(4):701-707.

30. Alamer M. The role of prolactin in thermoregulation and water balance during heat stress in domestic ruminants. Asian J Anim Vet Adv 2011;6(12):1153-1169.

31. Menéndez-Buxadera A, Molina A, Arrebola F, Clemente I, Serradilla JM. Genetic variation of adaptation to heat stress in two Spanish dairy goat breeds. J Anim Breed Genet 2012;129(4):306-315.

32. McManus C, Paludo GR, Louvandini H, Gugel R, Sasaki LCB, Paiva SR. Heat tolerance in Brazilian sheep: Physiological and blood parameters. Trop Anim Health Pro 2009;41(1):95-101.

33. Titto CG, Veríssimo CJ, Pereira AMF, Geraldo A de M, Katiki LM, Titto EAL. Thermoregulatory response in hair sheep and shorn wool sheep. Small Ruminant Res 2016;144:341-345.

34. Fadare AO, Peters SO, Yakubu A, et al. Physiological and haematological indices suggest superior heat tolerance of white-coloured West African Dwarf sheep in the hot humid tropics. Trop Anim Health Pro 2012;45(1):157-165.

35. Moberg GP. Biological response to stress: implications for animal welfare. In: Moberg, GP, Mench JA, editors. The biology of animal stress: Basic principles and implications for animal welfare. Wallingford: CABI; 2000:1-21.

36. da Silva WE, Leite JHGM, de Sousa JER, et al. Daily rhythmicity of the thermoregulatory responses of locally adapted Brazilian sheep in a semiarid environment. Int J Biometeorol 2017;61(7):1221-1231.

37. Fonseca VCF, Saraiva EP, Maia ASC, Nascimiento CCN, da Silva JA, Pereira WE, et al. Models to predict both sensible and latent heat transfer in the respiratory tract of Morada Nova sheep semiarid tropical environment. Int J Biometeorol 2017(5);61:777784. 
38. Macías Cruz U, Gastélum MA, Avendaño-Reyes L, Correa-Calderón A, Mellado M, Chay-Canul A, et al. Variaciones en las respuestas termoregulatorias de ovejas de pelo durante los meses de verano en un clima desértico. Rev Mex Cienc Pecu 2018;9(4):739753.

39. Cain III JW, Krausman PR., Rosenstock SR, Turner JC. Mechanisms of thermoregulation and water balance in desert ungulates. Wildlife Soc B 2006;34(3):570581.

40. Sejian V, Maurya VP, Naqvi SMK. Adaptability and growth of Malpura ewes subjected to thermal and nutritional stress. Trop Anim Health Prod 2010;42(8):1763-1770.

41. Matteri RL, Carroll JA, Dyer CJ. Neuroendocrine responses to stress. In: Moberg GP, Mench JA, editors. The biology of animal stress: Basic principles and implications for animal welfare. Wallingford: CABI; 2000:43-76.

42. Sejian V, Maurya VP, Kumar K, Naqvi SMK. Effect of multiple stresses on growth and adaptive capability of Malpura ewes under semi-arid tropical environment. Trop Anim Health Pro 2013;45(1):107-116.

43. Tsigos C, Chrousos GP. Hypothalamic-pituitary-adrenal axis, neuroendocrine factors and stress. J Psychosom Res 2002;53(4):865-871.

44. Mahjoubi E, Amanlou H, Mirzaei-Alamouti HR, et al. The effect of cyclical and mild heat stress on productivity and metabolism in Afshari lambs. J Anim Sci 2014;92(3):1007-1014.

45. Baumgard LH, Rhoads RP. Effects of heat stress on postabsorptive metabolism and energetics. Annu Rev Anim Biosci 2013;1(1):311-337.

46. Nelson EAS, Wong Y, Yu LM, Fok TF, Li K. Effects of hyperthermia and muramyl dipeptide on IL-1 $\beta$, IL-6, and mortality in a neonatal rat model. Pediatr Res 2002;52(6):886-891.

47. Morigny P, Houssier M, Mouisel E, Langin D. Adipocyte lipolysis and insulin resistance. Biochimie 2016;125:259-266.

48. Afsal, A, Sejian, V, Bagath, M, Krishnan, G, Devaraj, C BR. Heat stress and livestock adaptation: Neuro-endocrine regulation. Int J Vet Anim Med 2018;1(2):1-8.

49. Binsiya TK, Sejian V, Bagath M, Krishnan G, Hyder I, Manimaran A, et al. Significance of hypothalamic-pituitary-adrenal axis to adapt to climate change in livestock. Int Res J Agric Food Sci 2017;2(1):1-20. 
50. Vicente-Pérez A, Avendaño-Reyes L, Barajas-Cruz R, Macías-Cruz U, Correa-Calderón A, Corrales-Navarro JL, et al. Parámetros bioquímicos y hematológicos en ovinos de pelo con y sin sombra bajo condiciones desérticas. Ecosist Rec Agropec 2018;5(14):259.

51. Macías-Cruz U, Correa-Calderón A, Mellado M, Meza-Herrera CA, Aréchiga CF, Avendaño-Reyes L. Thermoregulatory response to outdoor heat stress of hair sheep females at different physiological state. Int J Biometeorol 2018;62(12):2151-2160.

52. Macías-Cruz U, Stevens JC, Correa-Calderón A, Mellado M, Meza-Herrera CA, Avendaño-Reyes L. Effects of pre-lambing maternal energy supplementation on postweaning productive performance and thermoregulatory capacity of heat-stressed male lambs. J Therm Biol 2018;75:7-12. 